\title{
Enthesopathy and involvement of synovio-entheseal complex in systemic sclerosis: an ultrasound pilot study
}

\author{
Riccardo Terenzi ${ }^{1, *}$, Rositsa Karalilova ${ }^{2, *}$, Gemma Lepri ${ }^{1}$, Cosimo Bruni ${ }^{1}$, \\ Silvia Bellando-Randone ${ }^{1}$, Mirko Manetti $\mathbb{D}^{3}$, Eloisa Romano ${ }^{1}$, \\ Daniela Melchiorre ${ }^{1}$, Jelena Blagojevic ${ }^{1}$, Yukai Wang ${ }^{1,4}$, Kamal Solanki ${ }^{5}$, \\ Alberto Moggi-Pignone ${ }^{6}$, Zguro Batalov ${ }^{2}$, Serena Guiducci ${ }^{1}$, Anastas Batalov $\mathbb{D}^{2}$ \\ and Marco Matucci-Cerinic ${ }^{1}$
}

\begin{abstract}
Objectives. SSc is a chronic autoimmune disease characterized by inflammation of the skin and multiple internal organs. Articular involvement is one of the main features of SSc, and typical hallmarks of SpA have been found in SSc patients. The aim of the present study was to estimate the prevalence of entheseal and synovio-entheseal complex (SEC) alterations in a cohort of SSc patients.

Methods. One hundred SSc patients and 25 healthy subjects were included in this cross-sectional study. The enthesis sites of lateral epicondylar common extensor tendons (CET) and the enthesis of the Glasgow Ultrasound Enthesis Scoring System were evaluated. SEC involvement was evaluated only at CET enthesis.

Results. In SSc, the Glasgow Ultrasound Enthesis Scoring System score was significantly higher (median 4.0, interquartile range 2.0-7.0) than in controls (median 1.0, interquartile range 0.0-3.0) $(P<0.0001)$. CET enthesis of SSc patients showed more frequent US B-mode alterations than that of controls $\left(\chi^{2}=11.47, P=0.0007\right.$ for size; $\chi^{2}=13.79, P=0.0002$ for cortical irregularity, $\chi^{2}=5.24, P=0.022$ for calcification/enthesophytes). Power Doppler US signal at CET enthesis was significantly more frequent in SSc patients than in healthy controls $\left(\chi^{2}=9.11, P=0.0025\right)$, as was the concomitant SEC involvement $\left(\chi^{2}=8.52, P=0.0035\right)$.

Conclusion. These data show that SSc patients frequently present US features of enthesopathy. Moreover, CET enthesopathy was correlated with SEC inflammation, suggesting that entheseal inflammation in SSc may share the same micro-anatomical targets as found in SpA.
\end{abstract}

Key words: systemic sclerosis, scleroderma, ultrasound, enthesopathy

\section{Rheumatology key messages}

- SSc patients frequently present US features of enthesopathy.

- Entheseal inflammation in SSc has the same micro-anatomical targets as those of SpA, including the synovioentheseal complex.

- Symptomatic enthesopathy may be underappreciated in SSc.

${ }^{1}$ Department of Experimental and Clinical Medicine, Section of Internal Medicine, University of Florence, and Division of Rheumatology, Azienda Ospedaliero-Universitaria Careggi (AOUC), Florence, Italy, ${ }^{2}$ Department of Internal Diseases, Medical University of Plovdiv, Clinic of Rheumatology, University Hospital 'Kaspela', Plovdiv, Bulgaria, ${ }^{3}$ Department of Experimental and Clinical Medicine, Section of Anatomy and Histology, University of Florence, Florence, Italy, ${ }^{4}$ Department of Rheumatology and Immunology, Shantou Central Hospital, Shantou, Guangdong, China,

${ }^{5}$ Rheumatology Department, Waikato Hospital, Hamilton, New Zealand and ${ }^{6}$ Department of Experimental and Clinical Medicine,
Section of Internal Medicine, University of Florence, and Division of High Complexity Medicine, Azienda Ospedaliero-Universitaria Careggi (AOUC), Florence, Italy

Submitted 11 March 2019; accepted 2 July 2019

${ }^{*}$ Riccardo Terenzi and Rositsa Karalilova contributed equally to this study.

Correspondence to: Riccardo Terenzi, Department of Experimental and Clinical Medicine, University of Florence, Largo Brambilla 3, 50134 Florence, Italy. E-mail: terenzi.ricca@gmail.com 


\section{Introduction}

SSc is a chronic autoimmune disease with a complex pathogenesis characterized by fibrosis and inflammation of the skin and multiple internal organs [1]. Despite the multisystemic organ involvement, at present there are no validated predictors of disease progression, which limits stratification of SSc patients and an early therapeutic approach [2].

Articular involvement is a frequent feature of SSc [3] as testified by cross-sectional analyses of the EULAR Scleroderma Trial and Research group (EUSTAR) database that revealed a prevalence of $28 \%$ as well as a potential role of joint and tendon involvement in predicting disease evolution [4,5]. Moreover, in SSc joint synovitis appears to be associated with disease activity and systemic inflammation, and is more likely to occur in the early stages of the disease $[4,5]$. US studies suggest that synovitis is one of the most common findings in SSc, affecting almost half of all patients $[6,7]$. Of note, the evidence that synovitis is commonly mild with a low-grade power Doppler US (PDUS) vascularization [6, 7] may result in the underestimation of SSc-related articular involvement. Furthermore, in SSc, joint involvement may be heterogeneous as patients may present tenosynovitis and synovitis, CTS and bursitis, as well as erosive changes [6]. Interestingly, the typical hallmarks of SpA, such as sacroiliitis, have been reported with a prevalence of $23 \%$ in a case series of SSc patients [8]. Tendon involvement is also common in SSc. In particular, tenosynovitis of the hand was found in $27 \%$ of SSc patients, showing a mixed pattern of inflammatory and fibrotic changes [6]. In addition, other studies suggested that even knee and ankle tendons without sheath are altered in SSc patients [9] and that enthesopathy is more frequent in SSc patients than in healthy subjects [10]. Collectively, the bulk of data suggest that there are SpA-like articular manifestations in SSc, but only one study focussed on the prevalence of entheseal alterations in SSc patients and none on the pathologic alterations of the synovio-entheseal complex (SEC) in this disease.

On these bases, the aim of the present study was to estimate the prevalence of entheseal and SEC alterations in a cohort of SSc patients.

\section{Methods}

\section{Patients}

One hundred SSc patients [16 male, 84 female, mean age 53.3 (16.6) years] fulfilling the 2013 ACR/EULAR SSc classification criteria [11] were included in this cross-sectional study. SSc patients were consecutively recruited from the outpatient clinic of the Division of Rheumatology of the Azienda Ospedaliero-Universitaria Careggi (AOUC), Florence, Italy and the Clinic of Rheumatology of the University Hospital 'Kaspela', Plovdiv, Bulgaria. None of the enrolled SSc patients had a previous diagnosis of musculoskeletal involvement or were treated with steroids at the time of the evaluation. Patients were categorized into two subsets, namely dcSSc (54 patients) and IcSSc (46 patients). The presence of clinically symptomatic enthesopathy in bone insertions of lateral epicondylar extensor tendons, quadriceps tendons, patellar ligament (at both patellar and tibial insertions), Achilles tendons and plantar fascia insertions on the calcaneus were defined as the presence of pain elicited by finger pressure on the aforementioned entheseal sites (i.e. entheseal tenderness). The presence of clinically symptomatic enthesopathy was evaluated by two rheumatologists (J.B. and S.B.-R.) blinded to patients' clinical features. Twenty-five healthy subjects [5 male, 20 female, mean age 46.9 (5.8) years] were used as the control population. The study was approved by the local institutional review boards at the AOUC, Florence, Italy and the University Hospital 'Kaspela', Plovdiv, Bulgaria. All patients provided written informed consent.

\section{US assessment}

US scanning of the entheses was performed by two rheumatologists trained in musculoskeletal US (R.T. and R.K.) and blinded to patients' characteristics and clinical features, with a MyLab 70XVG scanner (Esaote, Genova, Italy) equipped with a $6-18 \mathrm{MHz}$ linear transducer (Esaote). Each tendon was scanned twice in both longitudinal and transverse planes, and the scan images were stored. Each examination took about $20 \mathrm{~min}$. The enthesis sites of lateral epicondylar common extensor tendons (CET) and the entheseal insertions of the Glasgow Ultrasound Enthesis Scoring System (GUESS) [12] were scanned bilaterally. GUESS score was calculated for every subject, as well as the GUESS soft tissue score and GUESS bone score sub-items [12]. The following enthesis sites included in the GUESS score were assessed: superior pole of the patella (quadriceps tendon insertion), the inferior pole of the patella (patellar ligament origin), patellar ligament insertion at the tibial tuberosity, Achilles tendon and plantar fascia insertions on the calcaneus. Patients were placed in a supine position to assess the patellar and quadriceps entheses. The knee was placed in $70^{\circ}$ flexion to assess greyscale abnormalities. Then the patients were placed in a prone position with the feet over the end of the examination table for the assessment of Achilles tendon and plantar fascia entheses. The following greyscale US findings indicative of enthesitis according to GUESS were documented: thickening of the tendon insertion, bone erosions, bursitis and enthesophyte formation [12]. The thickness of the enthesis was measured at the insertion of the deeper tendon margin into the bone in a longitudinal axis (Fig. 1A). CET was evaluated with the patient sitting in front of the examiner with the elbow flexed and the forearm lying on the examination table. The following US findings compatible with enthesis alterations according to the OMERACT definition of enthesitis [13] were documented: hypoechogenicity, increased thickness of the tendon insertion, calcifications, enthesophytes, erosions and presence of PDUS (Fig. 1B and C). SEC involvement was evaluated only at CET enthesis. In SSc patients who presented a PDUS signal 
FIG. 1 Representative US images of joint and enthesis
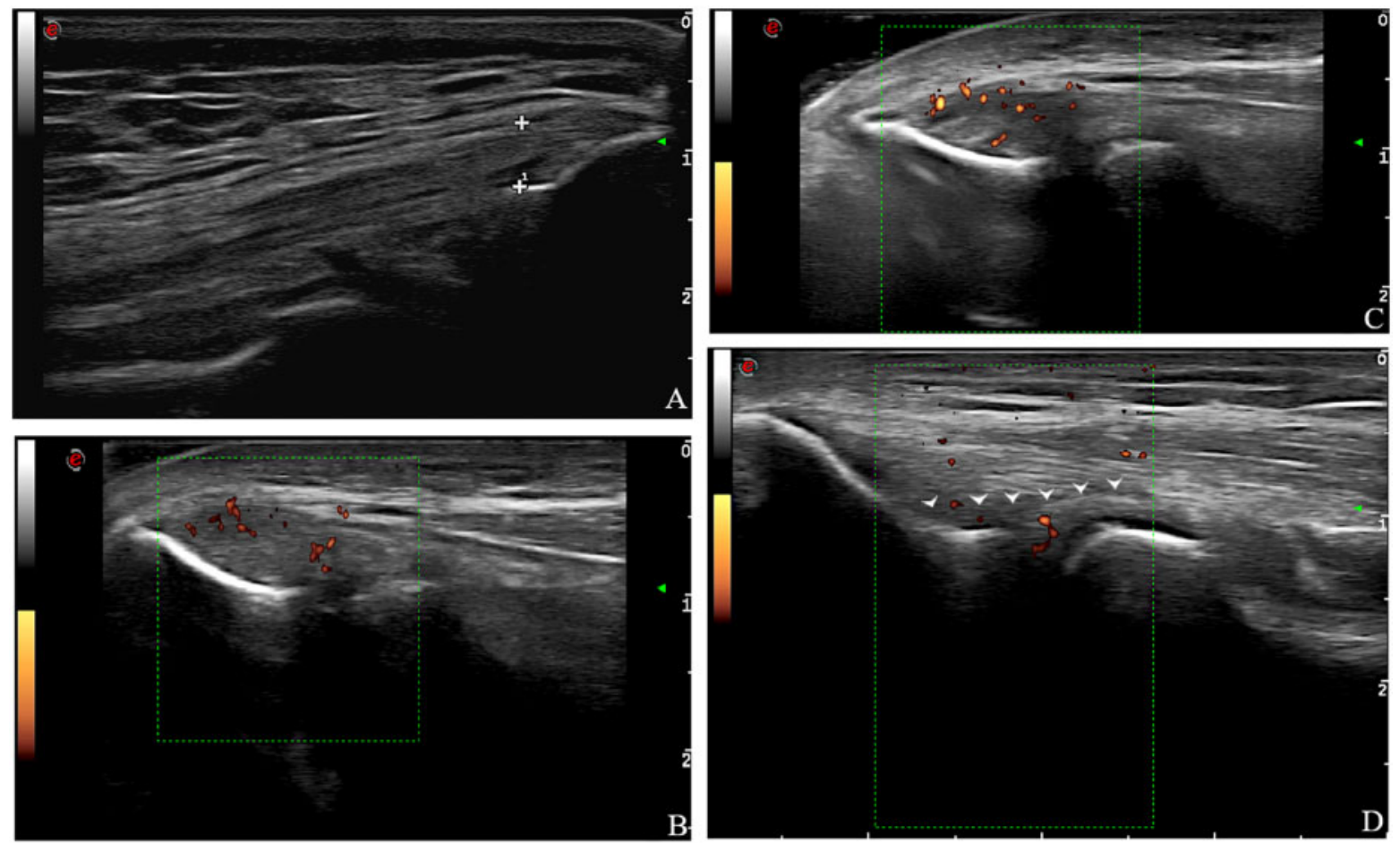

(A) Normal fibrillar echographic morphology of quadriceps tendon enthesis (beneath two white +). (B and C) Increased PDUS signals at lateral epicondylar CET enthesis in two representative patients with SSc. (D) Detail of the image shown in (C); note the increased PDUS signal at SEC level under the radial collateral ligament (below white arrowheads). PDUS: power Doppler US; CET: common extensor tendon; SEC: synovio-entheseal complex.

$\geqslant 1$ at CET closer than $2 \mathrm{~mm}$ to the bony surface, the involvement of SEC was evaluated at the epicondylar region. SEC involvement was defined as the presence of a PDUS signal $\geqslant 2$ in the elbow epicondylar synovial fold belonging to the lateral epicondyle enthesis [13], proximal to the annular ligament and inferiorly located to the bone insertion of CET and to the radial collateral ligament (Fig. 1D) [14]. To avoid PDUS reverberation artefacts, SEC PDUS signal was reported only if present in a different plane of CET PDUS signal and not just beneath it. PDUS settings were standardized with a Doppler frequency of $8-12.5 \mathrm{MHz}$, pulse repetition frequency of $750 \mathrm{~Hz}$, and a wall filter of 2. Gain was adjusted until the background signal was removed. PDUS signal was reported only if present in two orthogonal planes.

\section{Statistical analysis}

Statistical analysis was performed using SPSS V.24.0 package (Statistical Package for the Social Sciences, Chicago, IL, USA). To assess the normal distribution of data Shapiro-Wilk test was performed. Mann-Whitney $U$ test was employed for two-group comparisons of continuous variables, $\chi^{2}$ test for the comparison of frequencies and Spearman's rank correlation coefficient (rho) to examine the relationship between two continuous variables. All values are presented as median and interquartile range
(IQR). Values of $P<0.05$ were considered statistically significant.

\section{Results}

\section{GUESS score}

In SSc, the GUESS score was significantly higher (median 4.0, IQR 2.0-7.0) than in healthy controls (median 1.0, IQR 0.0-3.0) $(P<0.0001)$ (Fig. 2A). Moreover, both the GUESS bone and the GUESS soft tissue scores were significantly greater in SSc patients compared with controls (median 1.0, IQR 0.0-4.0 vs median 0.0, IQR 0.0-1.0, $P=0.005$ for GUESS bone; median 3.0, IQR 1.0-4.0 vs median 1.0, IQR 0.0-2.0, $P<0.0001$ for GUESS soft tissue) (Fig. 2B and C). No significant differences in GUESS score and its subitems (GUESS bone and GUESS soft tissue scores) were found between the dcSSc and IcSSc subsets.

\section{Lateral epicondyle enthesis alterations}

In CET entheses of SSc patients, all US findings compatible with enthesis alterations were detected according to the OMERACT definition of enthesitis: hypoechogenicity, increased thickness of the tendon insertion, calcifications, enthesophytes, erosions and presence of PDUS. CET enthesis of SSc patients showed more frequent US 
Fig. 2 (A) Results of GUESS score and $(\mathbf{B}, \mathbf{C})$ its subitems in $\mathrm{HC}$ and patients with SSc
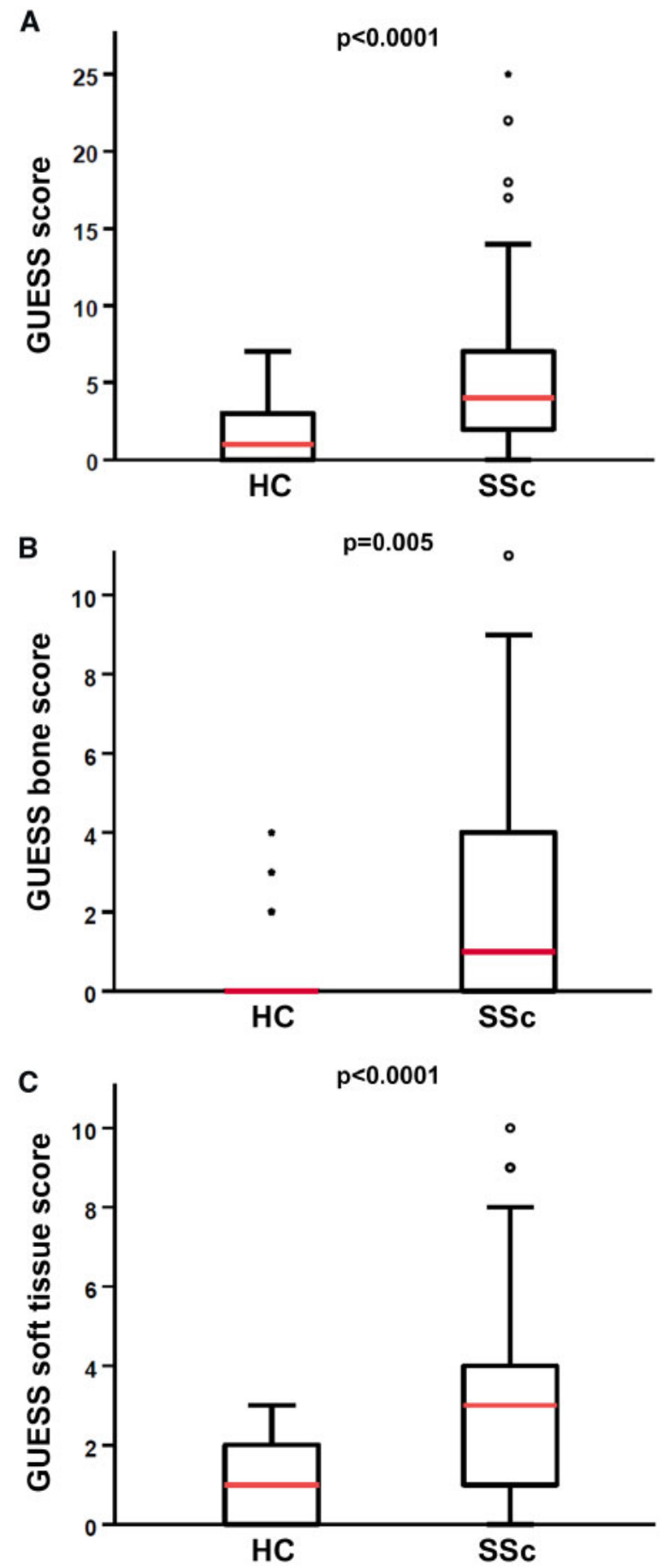

Data are shown as box plots. Each box represents the 25th to 75th percentiles. Red lines inside the boxes represent the median. Lines outside the boxes represent the 10th and the 90th percentiles. Circles indicate outliers, and asterisks indicate the extreme values. Mann-Whitney $U$ test was used for statistical analysis. GUESS: Glasgow Ultrasound Enthesis Scoring System; HC: healthy controls.
B-mode alterations than that of controls $\left(\chi^{2}=11.47\right.$, $P=0.0007$ for size; $\chi^{2}=13.79, P=0.0002$ for cortical irregularity; and $\chi^{2}=5.24, P=0.022$ for calcification/enthesophytes). PDUS signal at CET enthesis was significantly more frequent in SSc patients than in healthy controls $\left(\chi^{2}=9.11, P=0.0025\right)$, as was the concomitant SEC involvement $\left(\chi^{2}=8.52, P=0.0035\right)$. There was no significant difference in the frequency of B-mode alterations and the intensity of PDUS signals in CET enthesis when comparing the dcSSc and IcSSc subsets. Moreover, in SSc patients we observed a strong correlation between the presence of PDUS signal at CET enthesis and the concomitant SEC involvement (rho $=0.67, P<0.0001$ ).

\section{US findings and presence of clinically symptomatic} enthesopathy in SSc patients

In our SSc cohort, 38 patients (38\%) presented at least one clinically symptomatic enthesopathy in one of the two CET sites, while 63 patients (63\%) presented at least one clinically symptomatic enthesopathy in one of the entheseal sites of the GUESS score. In SSc patients, the GUESS score was positively correlated with the number of clinically symptomatic GUESS enthesis sites (rho $=0.72, P<0.0001$ ). As far as the correlation between clinically symptomatic CET enthesopathy and US features of CET is concerned, symptomatic SSc patients had a significantly higher frequency of US B-mode alterations than asymptomatic patients $\left(\chi^{2}=89.12, P<0.00001\right.$ for size; $\chi^{2}=20.34, P<0.00001$ for cortical irregularity; and $\chi^{2}=54.85, P<0.00001$ for calcification/enthesophytes). In addition, PDUS signal at CET enthesis was significantly more frequent in the SSc symptomatic group $\left(\chi^{2}=128.59, P<0.00001\right)$. Similar results were observed for SEC involvement $\left(\chi^{2}=69.15, P<0.00001\right)$.

\section{Calcinosis of the entheses}

In 3 out of 100 SSc patients (3\%, 2 IcSSc and 1 dcSSc) calcinosis was detected in at least one of the enthesis sites scanned. In two patients, a bilateral calcinosis of the lateral epicondyle enthesis was found, and one of them presented a calcinosis of the left plantar fascia enthesis. Calcinoses were located within the fibrillar structure of the tendon entheses with an involvement of the surrounding subcutaneous tissue. At US B-mode, calcinoses appeared as highly hyperechoic masses, with a round shape and with a posterior acoustic shadowing. The soft tissue around the calcinoses presented a high PDUS signal. All the enthesis sites with calcinosis were painful at examination.

\section{Discussion}

The data from the present study demonstrate that SSc patients commonly present US B-mode features of enthesopathy at GUESS entheseal insertions. This is further corroborated by the significantly higher GUESS score found in SSc patients than in healthy controls. Moreover, our analyses also revealed that US B-mode alterations and PDUS signals of CET enthesis are 
significantly more frequent in SSc than in controls. In particular, our findings clearly indicate that CET enthesopathy with concomitant SEC involvement can be frequently detected in SSc patients. Of note, in our SSc cohort we could also reveal a strong correlation between both GUESS and CET enthesis US findings and the presence of clinically symptomatic enthesopathy. Indeed, although our patients did not have a previous diagnosis of musculoskeletal involvement, many were found to have entheseal tenderness that correlated with the imaging findings, which suggests that symptomatic enthesopathy may commonly be underappreciated in SSc. Furthermore, $3 \%$ of our SSc patients presented calcinosis of at least one of the enthesis sites evaluated.

Articular involvement is a common feature of SSc and it is frequently underestimated [4, 5]. In SSc, synovitis is the most common joint manifestation, but specific SpA-like alterations have also been described [8, 10]. In this context, our data definitively confirm that enthesis alterations are common in SSc, affecting both upper and lower limbs. The current study is the first to assess SEC involvement in SSc patients. In particular, we focussed on CET enthesis and its associated SEC. Indeed, elbow lateral epicondyle enthesis is an easy site to scan and exhibits a well-defined synovial fold that, together with the radial collateral ligament and CET, contributes to the constitution of the SEC $[14,15]$. Despite the debate about whether enthesitis is a primary central lesion or a secondary process, clearly enthesitis is one of the hallmarks of SpA. In the effort to better understand the pathogenesis of SpA-related enthesitis, both magnetic resonance imaging and US studies have shown that entheseal changes were associated with pathological alterations of the adjacent bone and soft tissue, including synovial folds [16, 17]. Therefore, it has been argued that this micro-anatomical region should be considered as an 'entheseal organ', namely the SEC [17]. A large body of evidence indicates that SEC involvement is a fundamental process in the pathogenesis of SpA-related enthesitis, as biomechanical forces trigger an inflammatory cascade with cytokine production by infiltrating monocytes and lymphocytes in the adjacent synovial tissue, resulting into an articular inflammatory response [17]. In SSc, skin thickening and the changes of peritendineal structures [9] may represent a risk factor for an increased stress of the affected enthesis and, consequently, of SEC. In fact, it is well known that SSc patients are characterized by a significant skin thickening that may alter the distribution of mechanical forces on the underlying enthesis and SEC [18]. Interestingly, in SSc skin thickening is mainly reported in the early disease phases [18], which is when the articular involvement is predominant $[4,5]$. Moreover, SSc patients show a significant decrease of body fat mass in respect to healthy controls [19]. The loss of hypodermal adipose tissue may trigger a deregulated movement of the tendons and, consequently, a mechanical stress of entheseal structures. In vivo experiments also suggest that muscle strength has a key role in the tendon enthesis metabolism and functions [20]. Indeed, the absence of muscle loading and strength has been reported to cause histologically detectable defects in enthesis and fibrocartilage formation in mice [20]. In this regard, it is worth noting that SSc is strongly related to sarcopenia [19], which might affect enthesis metabolism and functions.

As far as the possible underlying pathogenetic mechanisms are concerned, it should be considered that the IL23/IL-17 axis is one of the main altered pathways in the development of SpA [21-24]. Furthermore, in animal models of SpA, SEC inflammation appears to be an IL23-dependent event and a fundamental step in the development of enthesitis [22]. The evidence that enthesopathy in SSc and SpA shares the same micro-anatomical involvement and features suggests a possible common pathogenetic cascade across these two different diseases. In SSc, it has been shown that IL-23 and IL-17 up-regulate collagen production in fibroblasts, a process that could be significantly reduced by an IL-17-blocking antibody $[25,26]$. On this basis, it can be speculated that an altered IL-23/IL-17 axis might play a role also in SScrelated enthesopathy. Further studies are necessary to decipher the pathomechanisms driving entheseal alterations in SSc.

To the best of our knowledge, this is also the first study reporting that in SSc calcinosis can painfully affect the entheseal region of the tendons. Of note, our imaging analyses clearly highlighted that enthesopathy can similarly affect patients with IcSSc and those with dcSSc.

In conclusion, here we show that SSc patients frequently present US features of enthesopathy. Strikingly, CET entheseal alterations were correlated with SEC involvement, suggesting that enthesopathy in SSc may share the same micro-anatomical targets of SpA. Considering the new wave of innovative treatments for SSc [27], we believe that disease-related enthesopathy should not be further overlooked. Future larger prospective studies are desirable to extend these preliminary results and to comprehensively investigate their clinical significance.

Funding: No specific funding was received from any funding bodies in the public, commercial or not-for-profit sectors to carry out the work described in this manuscript.

Disclosure statement: The authors have declared no conflicts of interest.

\section{References}

1 Varga J, Trojanowska M, Kuwana M. Pathogenesis of systemic sclerosis: recent insights of molecular and cellular mechanisms and therapeutic opportunities. J Scleroderma Relat Disord 2017;2:137-52.

2 Matucci-Cerinic M, Allanore Y, Czirják $L$ et al. The challenge of early systemic sclerosis for the EULAR Scleroderma Trial and Research group (EUSTAR) community. It is time to cut the Gordian knot and develop a prevention or rescue strategy. Ann Rheum Dis 2009;68:1377-80. 
3 Varjú C, Péntek M, Lóránd V et al. Musculoskeletal involvement in systemic sclerosis: an unexplored aspect of the disease. J Scleroderma Relat Disord 2017;2:19-32.

4 Avouac J, Walker U, Tyndall A et al. Characteristics of joint involvement and relationships with systemic inflammation in systemic sclerosis: results from the EULAR Scleroderma Trial and Research Group (EUSTAR) database. J Rheumatol 2010;37:1488-501.

5 Avouac J, Walker UA, Hachulla E et al. Joint and tendon involvement predict disease progression in systemic sclerosis: a EUSTAR prospective study. Ann Rheum Dis 2016;75:103-9.

6 Elhai M, Guerini H, Bazeli R et al. Ultrasonographic hand features in systemic sclerosis and correlates with clinical, biologic, and radiographic findings. Arthritis Care Res (Hoboken) 2012;64:1244-9.

7 Cuomo G, Zappia M, Abignano G et al. Ultrasonographic features of the hand and wrist in systemic sclerosis. Rheumatology (Oxford) 2009;48:1414-7.

8 Arslan Tas D, Yıldız F, Sakallı $\mathrm{H}$ et al. Sacroiliac joint involvement in systemic sclerosis. Int $\mathrm{J}$ Rheum Dis 2015;18:84-90.

9 Cuomo G, Zappia M, ludici M et al. The origin of tendon friction rubs in patients with systemic sclerosis: a sonographic explanation. Arthritis Rheum 2012;64:1291-3.

10 Kilic E, Kilic G, Akgul O et al. Presence of enthesopathy demonstrated with ultrasonography in systemic sclerosis. Mod Rheumatol 2015;25:731-6.

11 van den Hoogen F, Khanna D, Fransen J et al. 2013 classification criteria for systemic sclerosis: an American College of Rheumatology/European League Against Rheumatism collaborative initiative. Ann Rheum Dis 2013;72:1747-55.

12 Balint PV, Kane D, Wilson $\mathrm{H}$ et al. Ultrasonography of entheseal insertions in the lower limb in spondyloarthropathy. Ann Rheum Dis 2002;61:905-10.

13 Terslev L, Naredo E, lagnocco A et al. Defining enthesitis in spondyloarthritis by ultrasound: results of a Delphi process and of a reliability reading exercise. Arthritis Care Res (Hoboken) 2014;66:741-8.

14 Tsuji H, Wada T, Oda T et al. Arthroscopic, macroscopic, and microscopic anatomy of the synovial fold of the elbow joint in correlation with the common extensor origin. Arthroscopy 2008;24:34-8.

15 Jacobson JA, Chiavaras MM, Lawton JM et al. Radial collateral ligament of the elbow: sonographic characterization with cadaveric dissection correlation and magnetic resonance arthrography. J Ultrasound Med 2014;33:1041-8.

16 Benjamin M, Moriggl B, Brenner E et al. The "enthesis organ" concept: why enthesopathies may not present as focal insertional disorders. Arthritis Rheum 2004;50:3306-13.

17 Benjamin M, McGonagle D. The enthesis organ concept and its relevance to the spondyloarthropathies. Adv Exp Med Biol 2009;649:57-70.

18 Sedky MM, Fawzy SM, El Baki NA et al. Systemic sclerosis: an ultrasonographic study of skin and subcutaneous tissue in relation to clinical findings. Skin Res Technol 2013;19:e78-84.

19 Marighela TF, Genaro PDS, Pinheiro MM et al. Risk factors for body composition abnormalities in systemic sclerosis. Clin Rheumatol 2013;32:1037-44.

20 Schwartz AG, Lipner JH, Pasteris JD et al. Muscle loading is necessary for the formation of a functional tendon enthesis. Bone 2013;55:44-51.

21 Jacques $\mathrm{P}$, Lambrecht $\mathrm{S}$, Verheugen $\mathrm{E}$ et al. Proof of concept: enthesitis and new bone formation in spondyloarthritis are driven by mechanical strain and stromal cells. Ann Rheum Dis 2014;73:437-45.

22 Sherlock JP, Joyce-Shaikh B, Turner SP et al. IL-23 induces spondyloarthropathy by acting on ROR- $\gamma \mathrm{t}+$ CD3+CD4-CD8 - entheseal resident T cells. Nat Med 2012;18:1069-76.

23 Reveille JD. Genetics of spondyloarthritis-beyond the MHC. Nat Rev Rheumatol 2012;8:296-304.

24 Appel H, Maier R, Wu P et al. Analysis of IL-17+ cells in facet joints of patients with spondyloarthritis suggests that the innate immune pathway might be of greater relevance than the Th17-mediated adaptive immune response. Arthritis Res Ther 2011;13:R95.

25 Nakayama W, Jinnin M, Tomizawa $Y$ et al. Dysregulated interleukin-23 signalling contributes to the increased collagen production in scleroderma fibroblasts via balancing microRNA expression. Rheumatology (Oxford) 2017;56:145-55.

26 Yang $X$, Yang J, Xing $X$ et al. Increased frequency of Th17 cells in systemic sclerosis is related to disease activity and collagen overproduction. Arthritis Res Ther 2014;16:R4.

27 Khanna D, Distler JHW, Sandner P et al. Emerging strategies for treatment of systemic sclerosis. J Scleroderma Relat Disord 2016;1:186-93. 